\title{
Para la edición de las Coplas de Jorge Manrique: stemma y selección de variantes
}

\author{
For the edition of Jorge Manrique's Coplas: \\ stemma and selection of variants
}

Miguel Ángel Pérez Priego
UNED
mperez@flog.uned.es

ORCID iD: http://orcid.org/0000-0002-3571-2169

\begin{abstract}
RESUMEN: En este trabajo se presenta una nueva propuesta para la edición crítica de las Coplas sobre la muerte de su padre, de Jorge Manrique. Partiendo de la colación de testimonios lo más amplia y completa posible, se establece un nuevo stemma codicum con prevalencia de la tradición $\alpha$ y del manuscrito LB3. Ese stemma es el que debe guiar la selección de variantes, que arrojará una nutrida serie de lecciones hasta ahora casi nunca tenidas en cuenta por los editores del poema.
\end{abstract}

Palabras clave: crítica textual, edición, literatura del siglo $\mathrm{xV}$, cancioneros, poesía elegíaca, Jorge Manrique.

ABSTRACT: This paper presents a new proposition for the critical edition of the Coplas sobre la muerte de su padre, de Jorge Manrique. Based on the collation of the most comprehensive and complete number of copies, a new stemma codicum with prevalence of $\alpha$ tradition and LB3 is established. This stemma should guide the selection of variants, which reveal a large number of expressions and words so far rarely taken into account by the editors of the poem.

Keywords: textual criticism, literature of the $15^{\text {th }}$ century, songbooks, elegiac poetry, Jorge Manrique. 
A lo largo de las últimas décadas se han publicado muchas y valiosas ediciones de las Coplas sobre la muerte de su padre, de Jorge Manrique. Prácticamente todas y cada una de las casas editoriales han encomendado a un editor de solvencia la tarea de presentar el texto de la famosa obra (Caravaggi, 1984; Serrano de Haro, 1986; Beltrán, 1988, 1993 y 2013; Pérez Priego, 1990; Gómez Moreno, 2000; Morrás, 2003). Recogiendo las aportaciones de todos ellos y tratando de ofrecer ahora un planteamiento ecdótico más detallado y sistematizado, propongo unas nuevas bases de edición crítica. A partir de una relación y colación de testimonios lo más amplia y completa posible, centro mi propuesta en dos operaciones fundamentales del proceso de edición: el diseño del stemma codicum y la selección de variantes.

Las Coplas sobre la muerte de su padre, terminadas de componer en 1477, fueron recogidas pronto en algunos cancioneros manuscritos y en diversos impresos. Los cancioneros manuscritos fueron al menos cuatro, relativamente tempranos: el de Egerton (LB3), el del Escorial (EM6), el de Oñate-Castañeda (HH1) y el de Guillén de Segovia (MN19). Más tarde se copiaron en el manuscrito conservado del Cancionero de Baena (PN1) y mucho después en el cancionero de Palacio 617 (MP2). También tempranos fueron los primeros impresos, publicados en ediciones que contienen principalmente las obras de fray Íñigo de Mendoza, como la de la Vita Christi por fray Íñigo de Mendoça (¿Zaragoza, 1482?) $(82 * J M)$ o la del Cancionero de fray Íñigo de Mendoza (Zamora, Antón de Centenera, ca 1483?) (83*IM), así como poco después en el Cancionero de Ramón de Llavia (Zaragoza, Juan Hurus, 1484-88?) (86*RL). En la última década del siglo XV, seguían editándose las Coplas de Manrique con las obras de fray Íñigo de Mendoza $(92 \mathrm{VC}, 95 \mathrm{VC})$ y sólo varias décadas después fueron incluidas por fin en ediciones del Cancionero general (35CG, 40CG). Desde comienzos del XVI conocieron una larga serie de glosadores, como Alonso de Cervantes, Diego Barahona, Luis de Aranda, Rodrigo de Valdepeñas, Francisco de Guzmán, Jorge de Montemayor, Luis Pérez y Gregorio Silvestre.

La relación y descripción sumaria de esos manuscritos y ediciones, en orden a la tradición textual a que pertenecen y dejando ahora fuera las glosas, es la siguiente ${ }^{1}$ :

\section{LB3 Cancionero Egerton (British Library, Eg. 939)}

Manuscrito en papel y marcas de agua de finales 1470 y primeros 1480; en $4^{\circ}$, letra gótica cursiva; copista de origen castellano; copia de un original lle-

\footnotetext{
${ }^{1}$ Para los primeros pasos de la recensio, aparte las ediciones precedentes de las Coplas, se ha tenido en cuenta el trabajo de Beltrán (1987), así como sus sucesivas ediciones del texto de Manrique (1988, 1993 y 2013). Sobre el orden de las estrofas, se han aceptado las conclusiones de los artículos de Palumbo, (1983) y de Senabre (1983). Para la identificación de los testimonios, sigo el sistema de siglas de Dutton (1990-1991).
} 
vada a cabo en la corte de Navarra: contiene un conjunto de piezas diversas, muchas, como se ha dicho, de orientación proisabelina y otras de carácter consolatorio, religiosas y morales. Como pudiera interpretarse por las dos epístolas en prosa iniciales, irían dirigidas a la familia real de Navarra con motivo de la muerte de algunos de sus miembros en esos años de 1470; otras piezas se añadirían más tarde h. 1480. Las Coplas ocupan los fols, 15r-18v, con la siguiente rúbrica: "Coplas que fizo don jorje Manrique sobre la muerte del maestre de Santiago don Rodrigo Manrique su padre"; los últimos ocho versos están copiados de otra mano, aprovechando los blancos inferiores del folio. Han estudiado recientemente el cancionero Severin (2000) y Beltrán (2011). Reproducción digital del cancionero en el enlace:

$<$ http://www.cervantesvirtual.com/obra-visor/cancionero-del-siglo-Xv-manuscrito- $0 / \mathrm{html} />$.

82*JM Vita Christi por fray Íñigo de Mendoça [y otras coplas] (s.l., s.i., s.a., aunque posiblemente Zaragoza, 1482?)

Es la edición impresa más antigua de las Coplas; se encuentra en un incunable de la Vita Christi de fray Íñigo de Mendoza, que contiene también el Sermón de fray Íñigo y el Regimiento de príncipes de Gómez Manrique. Hay dos ejemplares conocidos del incunable, uno en la Biblioteca del Escorial, XII-17, y otro en la Biblioteca Comunale de Palermo, Esp-XI, F. 56, núm. 3; en aquel las Coplas se insertan entre el Sermón y el Regimiento, y en este van al final de todo. Están encabezadas por la siguiente rúbrica: "Dezir de don Jorje Manrique por la muerte de su padre". Los dos ejemplares tienen el mismo orden (o más bien desorden) de estrofas frente a toda la tradición: 112, 25-36, 13-24, 37-40. Aunque eso dio pie a especulaciones diversas sobre la composición del poema, hace tiempo quedó demostrado que esa alteración de estrofas es debida a un simple accidente material de transposición de hojas. Los textos de ambos ejemplares fueron reproducidos por Pérez Gómez (1958 y 1975), quien opina que proceden de una edición zaragozana anterior de la Vita Christi (Zaragoza, Pablo Hurus y Hans Planch, 1482?).

86*RL Cancionero de Ramón de Llavia (Zaragoza, Pablo Hurus, 1484-88?) Ejemplares en BNE, I-2567, I-2892.

Impreso en letra gótica, a dos cols., 98 fols. Dedicado a doña Francisquina de Bardají, esposa de Juan Fernández de Heredia, Gobernador de Aragón, el autor ha seleccionado "de muchas obras cathólicas puestas por coplas, las más esmeradas e perfectas". Son unos veinte poemas didácticos y morales, de poetas de todo el s. XV: Pérez de Guzmán, Mena, Mendoza, Jorge Manrique, Álvarez Gato, Gonzalo Martínez de Medina, Ervías, Gómez Manrique, Ruiz de Sevilla, Sánchez de Talavera y fray Gauberte. Las Coplas ocupan los fols. 73r-76, con el siguiente encabezamiento: "De don jorge manrique por la muerte de su padre". $<$ http://bdh-rd.bne.es/viewer.vm?id=0000108057\&page=1>.

HH1 Cancionero de Oñate-Castañeda (Harvard, Houghton Library, Ms.Esp. 97) Ms. letra gótica f. XV, papel y filigranas de h. 1480-1486; compilado posiblemente por el poeta jienense Pedro de Escavias. Contiene poesías de diversos 
autores, sobre todo, Pérez de Guzmán, Santillana, y Mena, también, Gómez Manrique, Costana, Diego de Burgos, fray Íñigo de Mendoza, Diego de San Pedro. Las Coplas ocupan los fols. 421v-424v: "Coplas de don jorge manrique a la muerte del maestre don rodrigo manrique su padre". Falta el fol. 425, por lo que el texto del poema queda incompleto, falto de las seis últimas estrofas. Índice y edición de los poemas inéditos en Uhagón (1900); descripción, estudio y reproducción de variantes textuales por Garcia (19781980). Edición de Severin e introducción de Garcia (1990), Reproducción digital casi completa en el enlace:

$<$ http://www.lluisvives.com/servlet/SirveObras/p227/0036739208806123991 0046/index.htm>.

MN19 Cancionero de Pero Guillén de Segovia (BNE, Ms. 4114)

Ms. de finales del s. XV, conservado en una copia del s. XVIII; se hubo de recopilar en parte por Pero Guillén en el núcleo cultural toledano en torno al arzobispo Alonso Carrillo. Las Coplas se hallan en fols.407-418; el poema es acéfalo y no comienza hasta la copla 10 .

$<$ http://bdh-rd.bne.es/viewer.vm?id=0000134993\&page=1>.

83*IM Cancionero de fray Íñigo de Mendoza (¿Zamora, Antón de Centenera, ca. 1483?)

Hay un ejemplar en la Biblioteca del Monasterio del Escorial, sign. 38-I-22, que describió Fernández Álvarez (1911) y, por sus caracteres tipográficos, asignó la impresión a Antón de Centenera, que ya había editado a fray Íñigo; otro ejemplar en la BNE, sign. I-897. Las Coplas se hallan en fols. 67r-70r: "Coplas que hizo don jorge manrique a la muerte del maestre de santiago don rodrigo manrique su padre".

$<$ http://bdh-rd.bne.es/viewer.vm?id=0000176297\&page=1>.

EM6 Cancionero de fray Íñigo de Mendoza (Biblioteca del Real Monasterio del Escorial, K-III-1)

Ms. en papel, letra fines XV; contiene diversas obras de fray Íñigo de Mendoza, las Coplas de los pecados mortales de Juan de Mena, la pregunta de Sancho de Rojas y las Coplas de Jorge Manrique, que ocupan los fols. 215v-225r: "Coplas que fizo don jorge manrique a la muerte del maestre de santiago don rodrigo manrique su padre". Con todas esa piezas van encuadernadas también algunas coplas impresas de fray Ambrosio Montesino y obras en prosa, como el Reportorio de los tiempos de Andrés de Li y un tratado de astrología de Bernardo de Granollachs; lo estudió y describió Fernández Álvarez (1904).

PN1 Cancionero de Baena (Bibliothèque Nationale de France, Esp. 37)

Las Coplas se hallan al final del texto conservado, ocupando los fols. 203r-205v, en distinto papel, de hacia 1500 , y en letra procesal de comienzos del s. XVI (algún fragmento, vv. 181-312, en letra cortesana algo más cuidada), diferente de la utilizada hasta ese lugar por los copistas; llevan al frente la rúbrica: "Co- 
plas que fizo don jorge manrique a la muerte del maestre de Santiago don Rodrigo Manrique su padre". Es copia bastante fiel de $83 * I M$, del que se aparta en media docena de lecciones para seguir a $86 * R L$ o a $82 * J M$.

$<$ http://gallica.bnf.fr/ark:/12148/btv1b8452204d/f15.planchecontact.r= cancionero.langFR $>$.

92VC Fray Íñigo de Mendoza, Vita Christi [y otros poemas] (Zaragoza, Pablo Hurus, 1492).

No se conserva ejemplar, pero sí una copia del s. XVIII en el manuscrito MN46 [BNE, Ms. 18183], fols. 251r-260v: "Coplas que hizo don Jorge Manrique a la muerte del Maestre de Santiago don Rodrigo Manrique su padre". Ha estudiado el manuscrito y ha transcrito el texto Díez Garretas (2010).

95VC Fray Íñigo de Mendoza, Vita Christi [y otros poemas] (Zaragoza, Pablo Hurus de Constancia alemán, 10.X.1495).

Hay un único ejemplar en Roma, Biblioteca Universitaria Alessandrina, INC 382. Cerrón Puga (2012) ha estudiado ese fondo bibliográfico. Las Coplas de Jorge Manrique se hallan en fols. 109r-112r: "Coplas que hizo don jorge manrique a la muerte del maestre de santiago don rodrigo manrique su padre". El cancionero ML1 (ms. M1-1-13 de la Fundación Lázaro Galdiano, fechado en 1498) parece copia de este $95 \mathrm{VC}$, pues concuerda con su contenido, reproduce la tabla y coloca en el mismo lugar las Coplas, aunque estas ya no las copia. Hubo una edición anterior del incunable, en el mismo lugar e imprenta, en 1492 (92VC), ya citado.

35CG Cancionero general en el qual se han añadido agora de nuevo en esta última impressión muchas cosas buenas: ha sido con diligencia corregido y emendado (Sevilla, Juan Cromberger, 1535, 2 de abril)

40CG Cancionero general en el qual se han añadido agora de nuevo en esta última impressión muchas cosas buenas... (Sevilla, Juan Cromberger, 1540, 20 de noviembre)

Son las primeras ediciones del Cancionero general que recogen las Coplas de Manrique, fols. 200v-203v: "Coplas que hizo don jorge manrique a la muerte del maestre de santiago don Rodrigo manrique su padre". Siguen mayoritariamente las lecciones de $83 * I M$, aunque ya es muy intensa y abundante la contaminación de $82 * J M$ y sus descendientes. Llevan a continuación la "Adición hecha por Rodrigo Osorio sobre dos coplas que hallaron al señor don Jorge Manrique en el seno quando lo mataron", con esas dos coplas ("O mundo por que nos matas" y "Es tu comienzo lloroso") más varias coplas de Rodrigo Osorio.

MP2 Cancionero de poesías varias (Madrid, Real Biblioteca, Ms. 617). Poesías de los ss. XV y XVI, terminado de recopilar hacia 1570. Las Coplas ocupan los fols. $211 \mathrm{r}-215 \mathrm{r}$, en una sección intermedia, precedidas de las $\mathrm{Co}$ - 
plas de Mingo Revulgo y de los Proverbios de Santillana. Al propio texto de las Coplas manriqueñas, tras la c. XXIV, incorpora las dos publicadas en $35 C G$ como adición de Rodrigo Osorio. Han defendido la autenticidad de esas dos coplas José J. Labrador, C. Ángel Zorita y Ralph A. Difranco, (1985), autores también de la edición y estudio del cancionero (1986).

LNP Lisboa, Biblioteca Nacional de Portugal, Mss. Reservados COD 11353. Texto copiado hacia 1545. En los fols. 97-100 copia las Coplas de Manrique, aunque incompletas (faltan cs. 27-35 y 40-42) y desordenadas, quizá por haber sido copiadas de una edición impresa desencuadernada, con abundantes lusismos. Ha sido estudiado por Hock (1987).

Llevada a cabo la colación de variantes y el cotejo de testimonios, de sus convergencias y divergencias, y tras detectar y valorar los errores comunes y ciertos, los auténticos loci critici, podemos establecer los agrupamientos de testimonios de la manera siguiente.

\section{LA TRADICIÓN $\alpha$ FRENTE A LA TRADICIÓN $\beta$}

Advertimos que existen dos tradiciones o ramas en la transmisión del poema. Una estaría representada principalmente por los testimonios LB3 82*JM $86 * R L$ (a los que se añadirían $H H 1$ MN19), que llamaríamos $\alpha$, y la otra constituida fundamentalmente por $83 * I M$ EM6 y la copia de $P N 1$, que llamaríamos $\beta$. En los márgenes de ese esquema quedaría un grupo de testimonios tardíos, que presentan un texto fuertemente contaminado por las dos tradiciones, serían esencialmente: $92 \mathrm{VC}$ 95VC 35CG 40CG MP2.

\section{La tradición $\beta$}

Los testimonios que configuran la rama $\beta$, presentan numerosas lecciones divergentes de la rama $\alpha$. Unas son claros errores, como (las variantes de $\beta$ van en la primera columna y las de $\alpha$ en la segunda):
v. 57 y allegamos / y llegamos,
v. 97 dezimos / dezidme,
v. 335 camelio / camilo,
v. 344 muchos cavalleros y cavallos / muchos moros y c.,
v. 420 padescera / perescedera,

Otras, si no errores, pudieran considerarse variantes adiáforas: 
v. 93 que contecen / que acaescen,

v. 120 se sostienen / se mantienen,

v. 251 fuéronle sino pesares / qué fueron sino pesares,

v. 399 con (su) halago / sin (y su) falago.

v. 471 olvidados / conservados.

Y muchas otras son variantes gramaticales (fonéticas, morfológicas o sintácticas), como:

v. 20 a de durar / que ha de durar, v. 26 el mar / la mar, v. 67 el hijo / aquel hijo, v. 124 ni les pidamos / no les pidamos, v. 149 es la celada / la celada, v. 150 a nuestro / nuestro, v. 234 que le siguió / le siguió, v. 256 a los grandes y medianos / que a los g. y m., v. 261 tan alta / tan alto, v. 271 y sus muy claras hazañas / y las sus c. h., v. 274 que si tú cruda, quando si c. / quando tú c., v. 329 theodorio / theodosio, v. 359 muy más tierra / más tierra, v. 399 con su halago, con halago / y su h., sin falago.

Aunque los testimonios de esta rama forman una clara y unitaria tradición textual, coincidentes en la mayoría de sus lecciones y errores, resultan también diferentes entre sí con diversas lecciones privativas. Lecciones privativas de $83 * I M$ frente al resto de la tradición son, por ejemplo:

v. 49 es este mundo / este mundo es,

v. 416 no eternal / no es eternal.

Errores privativos de EM6 son:

v. 22 dura / duró,

v. 134 y alleguen / y lleguen,

v. 201 que tenían / que tañían,

v. 305 maestre / maestro

En esta misma rama se colocaría el ya más tardío testimonio $P N 1$, coincidente en casi todos sus versos con $83 * I M$, al que sigue fielmente, apartándose incluso de los errores de EM6 (v. 49 es este mundo / este mundo es, v. 134 y lleguen / y alleguen, v. 305 maestro / maestre). No obstante, en varios lugares presenta lecciones propias de la tradición $\alpha$, que corrigen a $\beta$ : v. 97 Dezidme / Dezimos, 149 la celada / es la celada, v. 214 sido / seído, v. 260 que tan alto / que tan alta, v. 335 camilo / camelio, 344 moros y cavallos / cavalleros y cavallos, v. 420 pereçedera / padesçera. Lo más probable es que para estos casos haya tenido también a la vista un testimonio de $\alpha$, quizá $86 * R L$ o quizá uno de los pliegos sueltos $82 * J M$. 


\section{La tradición $\alpha=L B 3+\gamma$}

En la otra, en la rama $\alpha$, el testimonio más individual e independiente es $L B 3$, el cual presenta muchas lecciones particulares; unas son claros errores:

v. 107 a la hedat / all arrabal,

v. 331 marco alixandre / aurelio a.,

v. 335 canino / camilo,

v. 353 fijos y criados / hermanos y criados,

transposición de los vv. 379-80,

v. 417 duradera / verdadera,

v. 419 infernal / temporal,

v. 442 una esperanza / buena e.,

y otras las calificaría provisionalmente de variantes equipolentes:

v. 140 los plazeres / los deleites,

v. 174 leemos y oímos / oímos y leímos,

v. 203 ropas trenpadas / ropas chapadas,

v. 406 fazedla de la virtud / esfuércese la v.,

v. 443 vida verdadera / vida tercera,

v. 444 cobraréis / ganaréis.

En esa misma rama $\alpha, 82 * J M$ manifiesta también numerosas lecciones y errores propios, como la alteración del orden estrófico, que adelanta las coplas XXV-XXXVI después de la XII, y las diferencias en los siguientes versos:

v. 40 aficiones / ficciones,

v. 53 este camino / esta jornada,

v. 103 las manos / las mañas,

v. 116 varios y abatidos / baxos y a.,

v. 158 que leemos / que vemos,

v. 203 japadas / chapadas (trempadas),

v. 236 levole / metiole,

v. 270 y los pones / y traspones,

v. 283 japada / chapada,

v. 287 lo pasas de claro en claro / todo lo pasas de claro,

v. 293 tanto fermoso / tanto famoso,

v. 415 vida y honor / vida de honor,

v. 419 que la corona temporal / que la otra t.,

vv. 460-61 juntaste divinidat / a una cosa tan vil $<>$ Tú que a tu divinidad /

v. 478 y aunque la vida murió / que aunque la vida perdió. juntaste cosa tan vil,

Este testimonio $82 * J M$ posee al menos un error conjuntivo con $L B 3$, una haplografía, en este caso: 
v. 198 de amores / de amadores,

que probablemente estuviera en el subarquetipo $\alpha(L B 3+\gamma)$, pero que ya corregiría por conjetura $86 * R L$. Algo semejante ocurre en el

v. 323 marco tulio / m. atilio,

que indica que el error estaría ya en $\alpha$ (también estaba en $\beta$ ) y corregirían $86 * R L, H H 1$ (Autilio) y MN19 (Atilio).

Muestra asimismo errores conjuntivos con $86 * R L$, por lo que se reclamaría un subarquetipo $\delta$ :

ambos desplazan la copla VII detrás de la XIII,

leen erróneamente los versos:

v. 19 nadi no (nadino) / nadie no,

v. 103 las manos $(82 * J M)$ las maneras $(86 * R L)$ / las mañas: en ambos casos se trata de un mal entendimiento y desarrollo de lo que se toma por abreviatura de la $\tilde{n}$ de mañas;

$\mathrm{y}$ ofrecen lecciones comunes y divergentes:

v. 13 Pues si / Y pues,

v. 56 andamos mientra / a. quando,

v. 112 se pierde / se sume,

v. 173 aunque oímos e leemos / a. leemos y o., a. o. y leímos,

Hay asimismo diversas lecciones comunes entre $L B 382 * J M 86 * R L$, como descendientes de $\alpha$ :

v. 20 que ha de durar / ha de durar,

v. 40 que traen / porque traen,

v. 62 usásemos / usáremos (usáramos),

v. 94 por calidad / por su c.,

v. 385 después de puesta / después que puso.

$86 * R L$ lee con $L B 3$ frente a los demás en:

v. 33 y llegados / allegados,

v. 174 estorias / victorias.

Y por supuesto $86 * R L$ tiene errores propios:

v. 231 le fizieron / se llamó,

v. 234 le siguieron / le siguió,

v. 245 mas solo / sino solo,

v. 329 verdosio / teodosio, 
Por último, $82 * J M$ ha podido contaminar a $83 * I M$ (y de ahí a EM6):

v. 33 allegados (alleguados) / y (ô) llegados,

v. 121 nos dexan / nos dexen,

v. 164 que a papas y e. / que papas y e.,

v. 322 un archiano / aureliano.

En cuanto a los manuscritos $H H 1$ y $M N 19$, presentan peculiaridades propias, aparte de lo incompleto de su texto, uno inacabado y otro falto del comienzo, por lo que sólo tienen en común 288 versos. $H H 1$ presenta numerosas lecciones y errores singulares:

v. 46 viniendo / viviendo,

v. 176 aquel tiempo / aquel siglo,

v. 236 echole / metiole,

v. 256 que a los baxos / que a los grandes,

v. 287 todo lo llevas / todo lo pasas,

v. 289 aquel de buenos amigo / aquel de b. abrigo,

v. 322 el troyano / aureliano,

v. 361 ganó / pintó.

También presenta errores privativos MN19:

v. 141 corporales / temporales,

v. $224 \mathrm{om}$.

Pero ambos comparten lecciones características de la tradición $\alpha$ y no $\beta$ :

v. 131 ni estar estable / ni ser e.

v. 186 truxieron / traxieron

v. 260 que tan alto / que tan alta

v. 344 moros y cavallos / cavalleros y cavallos

HH1 puede leer con LB3:

v. 301 que amigo de amigos / amigo de sus

con $L B 382 * J M$ :

v. 148 que son sino / no son sino,

v. 251 que fueron / fueronle;

o con $L B 3$ y $86 * R L$ :

v. 33 y llegados / allegados. 
Hay lecciones de la rama $\beta$ que, como esta última, parecen transferidas desde $86 * R L$ :

v. 14 ses ido / es ido,

v. 26 la mar / el mar,

v. 67 aquel fijo / el hijo,

v. 93 que acaescen / que contecen.

Varias de estas lecciones son compartidas también por $M N 19$, lo que podría indicar contaminación de $86^{*} R \mathrm{~L}$ a $\varepsilon$ :

v. 274 quando tú cruda / que si tú cruda,

v. 323 autilio (atilio) / tulio,

v. 335 camilo / camelio;

como asimismo revelarían otras lecciones comunes de $86 * R L$ y $M N 19$ :

v. 186 truxieron / traxieron,

v. 174 estorias / victorias,

v. 251 que le fueron / que fueron (fuéronle),

v. 322 aureliano (abreliano) / un archiano,

v. 458 forma servil / forma cevil.

Y otras las mantienen ellos dos, HH1 y MN19, en común, (confirmando una vez más la existencia del subarquetipo $\varepsilon$ ):

v. 158 que vedes / que vemos,

v. 204 que vestían / que traían,

v. 214 sido / seído.

\section{LOS TESTIMONIOS 92VC Y 95VC}

Por lo que se refiere a las demás ediciones, las zaragozanas de Hurus, $92 V C$ y $95 V C$ siguen principalmente el texto de $\alpha$, si no exactamente a través de la anterior impresión zaragozana conocida $82 * J M$ (que está llena de errores propios), sí a través de su ascendiente (otra perdida impresión salida de las mismas prensas). Con aquella coinciden en el desplazamiento de la copla VII detrás de la XIII y en no pocas lecciones características (v. 41 que traen, v. 56 andamos mientra vivimos, v. 93 que acaescen, v. 120 se mantienen, v. 270 y los pones, v. 274 quando tú cruda, v. 386 después de puesta). No obstante, hay muchos lugares en los que leen con $83 * I M$, en un alto grado de contaminación, que viene a indicar más bien la creación de un texto mixto a partir de $82 * J M$ y 83*IM que difundirá la imprenta desde estos últimos años del siglo XV (v. 34 allegados, v. 49 es este mundo, v. 97 dezimos, v. 384 cavalleros y cavallos). 
Aparte de esa serie de lecciones cruzadas, hay algún caso en que resulta revelador ese grado de fusión de textos: en el v. 420 leen padescedera, conjugando la errónea padescera $(\beta)$ con la correcta perescedera $(\alpha)$, o el v. 335 gamelio, frente a camilo $(\alpha)$ y camelio $(\beta)$. Por lo demás, hay que decir que $92 \mathrm{VC}$ y $95 \mathrm{VC}$ presentan idéntico texto con muy raras discrepancias entre los dos impresos (alguna errata tipográfica por cambio de caja, subda por duda, o adición de signo sobiranos por sobirnos, o por separación de palabras, su vida por suvida). Hay dos lecciones discrepantes más difíciles de explicar: v. 113 se sume en $92 \mathrm{VC}$ frente a se pierde en 95VC, y v. 471 olvidados / conservados. Puede que se trate de innovaciones introducidas en la copia moderna del impreso: que el copista del s. XVIII (por el único que conocemos 92VC) haya querido enmendar el texto con una lección que había oído o visto en otros textos. Es decir, que la contaminación se haya producido, no en el s. XV, sino en el XVIII.

En las ediciones sevillanas de Cromberger del Cancionero general, 35CG y $40 C G$, las Coplas reflejan esa misma situación y texto mixto: el desplazamiento de la copla VII y lecciones varias tanto de $82 * J M$ como de $83 * I M$ y no otras, la mayoría de las veces ya a través del texto contaminado de $92 \mathrm{VC} 95 \mathrm{VC}$. Las dos, 35CG y 40CG, como quedó dicho, llevan a continuación del texto manriqueño la "Adición” de Rodrigo Osorio.

\section{EL TESTIMONIO MP2}

Algo parecido cabe decir del texto tardío que transmite $M P 2$, con predominio de lecciones características de $82 * J M$ y $86 * R L$ (ses ido, que a de durar, que traen, este mundo es, andamos mientras, que acaecen, dezidme, se pierde, se mantienen, sus historias, que le fueron, teodosio, a esta vida de onor, perecedera, conservados) y en menor número de $83 * I M$ (allegados, ni ser estable, oímos y leímos, después que puso, con alago), y alguna lección propia significativa, que viene a recuperar lecciones singulares de testimonios más antiguos: v. 322 en sus brazos un troyano (en la que coincide parcialmente con $H H 1$, pero en ninguna más de las lecciones singulares de este), v. 406 esfuerce vuestra virtud, v. 443 que la otra vida eterna / ganareis (donde tampoco habla de la vida tercera). No obstante, lo más característico de este $M P 2$ es la incorporación, tras la c. XXIV, de las famosas y discutidas dos coplas ("Es tu comienzo lloroso", "O mundo, pues que nos matas") que, como acabamos de decir, traían las ediciones de 1535 y 1540 del Cancionero general, a continuación del texto de las Coplas, a nombre de Diego Osorio con la rúbrica "Adición hecha por Rodrigo Osorio sobre las coplas que hallaron al señor don Jorge Manrique en el seno quando lo mataron"2.

\footnotetext{
${ }^{2}$ Estas dos coplas procederían, en realidad, de la tradición glosística y, en concreto, de la glosa de Alonso de Cervantes (Lisboa, 1501), que, aunque sigue la línea de $83 * V C$ (con numerosos
} 
El resultado de este análisis de los testimonios y sus agrupamientos textuales, que hasta aquí hemos llevado a cabo, podríamos representarlo en el siguiente cuadro de relaciones entre ellos, es decir, en el siguiente stemma:

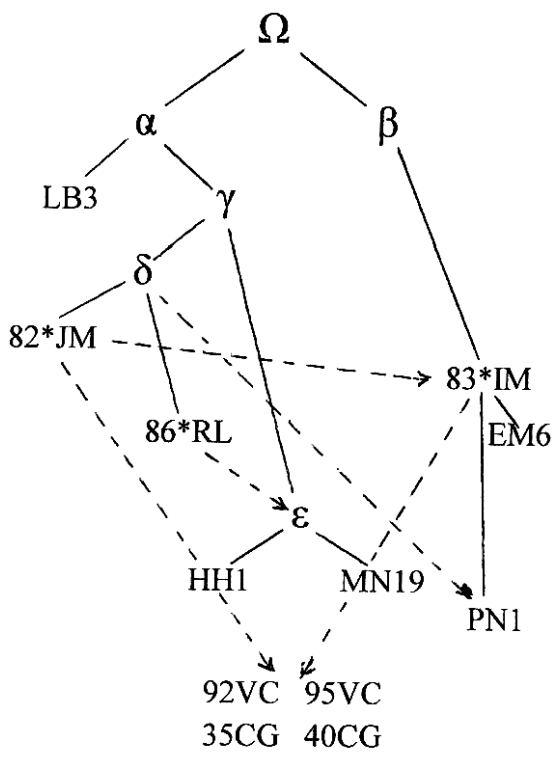

MP2

Tal es el esquema en que se nos muestran relacionados los distintos testimonios en el proceso de transmisión de las Coplas, el stemma, que habrá de guiarnos ulteriormente en las operaciones de la constitutio textus. Como se ve, sin embargo, el resultado es el del habitual stemma bipartito, que hace imposible la pura elección mecánica de variantes. Son dos ramas, con testimonios manuscritos e impresos, una más poblada que otra, pero ambas muy activas en virtud de la difusión que alcanzan sus testimonios impresos (los zaragozanos de

errores y alteraciones, y alguna lección contaminada de $82 * J M$ ), actúa con bastante libertad ante el texto manriqueño. Aparte de glosarlo copla a copla libremente, lo fragmenta materialmente y distingue como cuerpo aparte las coplas XXV-XXXIII, que corresponden al epicedio de don Rodrigo, que ya no glosa, pero sí encabeza con una nueva estrofa con la rúbrica "Copla sobre todos los que alaban a su padre. Don Jorge". De la misma manera, para cerrar toda la parte inicial de la obra, coplas I-XXIV, de reflexión moral sobre la muerte, entiendo decidiría añadir dos nuevas coplas meditativas como remate. De todos modos, no es ahora mi propósito entrar en esta parte de la tradición que constituyen las glosas, puesto que plantean un problema más de difusión que de transmisión del texto de las Coplas. 
Hurus y el cancionero de Llavia, por un lado, y el zamorano de Centenera por otro). Eso propició que en los textos de testimonios más tardíos se produjera una fuerte contaminación, como consecuencia del cruce de aquellas dos líneas de procedencia.

A la vista de todo, es claro que no puede postularse un texto más autorizado de las Coplas. Sin embargo, a la hora de continuar en busca del texto crítico, creo que, por su situación en el stemma, habría que contar con LB3 como firme candidato a texto base y sobre él corregir errores y seleccionar variantes. Es un testimonio que se sitúa en el plano alto del stemma, por tanto, más próximo al original, y no es un testimonio aislado sino que se agrupa con el subarquetipo $\gamma$, bajo el que a su vez se agrupan $82 * J M$ y $86 * R L$ y $H H 1$ y $M N 19$, a través de los interpuestos $\delta$ y $\varepsilon$ respectivamente. De manera que $L B 3$ tiene el mismo valor estemático que $\gamma$, es decir, que $82 * J M+86 * R L$ y $H H 1+M N 19$. Por lo demás, ese testimonio presenta algunos datos externos de mayor interés, como son la fecha de copia (derivada de la antigüedad del papel) o el carácter seguramente más conservador del entorno en que se copia. Los testimonios impresos conocidos son, en cambio, de fecha algo posterior y más inclinados a la innovación y alteración del texto.

Pues bien, en esa tarea que decíamos de selección de variantes, lo primero que constatamos es la gran abundancia de variantes lingüísticas o gramaticales que arroja la confrontación de testimonios en todo el proceso de transmisión de las Coplas. La mayoría de las veces son lecciones indiferentes, a partir de las distintas soluciones que ofrecía la lengua para un mismo o semejante fenómeno expresivo. Ocurren así numerosas variantes morfológicas, debidas, por ejemplo, a la elección del plural por el singular: v. 340 guerras a moros / guerra a los m.; al uso u omisión del artículo: v. 240 echaste el agua / echaste agua; a la variación en el uso de preposiciones: v. 164 que papas y emperadores / que a papas y e., v. 260 que en tan alto / que tan alto, v. 399 sin falago / con su halago; o en el uso de conjunciones: v. 41 que traen / porque traen, v. 208 y quan falaguero / quan f., v. $361 Y$ estas sus viejas istorias / Estas sus v. i., v. 368 y méritos y ancianía / por m. y a., v. 380 el nuestro rey / pues nuestro rey, v. 383 en Castilla / y (si) en C., v. 299 pues que todo el mundo sabe / pues el mundo t. s., v. 421 que el vivir / el vivir, 427 que los buenos / mas los buenos. Ocurren también en la terminación del adjetivo: v. 11 qualquier tiempo pasado / qualquiera, qualquiere, v. 146 trabajosa / trabajada; en el uso del posesivo: v. 94 por calidad / por su calidad, v. 271 y las sus / y sus muy; o de los ponderativos: v. 293 tanto famoso / tan famoso, v. 463 los grandes tormentos / tan g. $t$. La alternancia ocurre igualmente en la persona del verbo, en el tiempo o en la elección de la forma verbal más arcaica o más moderna: v. 40 no curéis / no curo, v. 62 usásemos / usáremos / usáramos, v. 214 seído / sido, v. 257 troxieron / truxieron / trayeron. 
Puede darse también alternancia en el orden sintáctico, más o menos grave y significativa: v. 286 quando tú vienes irada / que si tú v. airada, donde se introduce una oración temporal; v. 379-80 Si de las obras que obró, / el nuestro rey natural / fue servido, / dígalo el de Portugal $<>$ Pues nuestro rey natural, / si de las obras que obró..., donde el cambio de orden sintáctico deja entender más claramente el sentido, pero atenta contra el orden de rima ( $a b c b a c$ en lugar de $a b c a b c$ ).

Todas estas son variantes que nos encontramos vertidas en los distintos testimonios, que casi siempre se explican por las propias posibilidades de la lengua y que no comportan por sí mismas error. Por eso, porque no son claros errores, no puede establecerse sobre ellas la ordenación estemática. No indican que un testimonio esté copiado de otro, pues el copista de cada uno de ellos puede haber hecho la misma elección de manera independiente, puesto que estaba en las posibilidades del hablante. De todos modos, la variante linguiística puede dejar de ser indiferente si es contraria a la regularidad métrica (v. 11, v. 94 , v. 146) ${ }^{3}$ y, muchas veces, cuando se produce en el orden sintáctico e introduce matiz significativo: v. 13 Y pues vemos lo presente / Pues si vemos, v. 286 quando tú vienes / que si tú vienes. Los dos criterios pueden entrar en conflicto, como en los vv. 379-80. En último caso, la selección de este tipo de variantes vendrá dada por el testimonio que sea elegido como texto base, que será el que sigamos en la materialidad del texto.

Pero hay otras variantes de más peso, en las que quisiera fijar ahora la atención. Son variantes de sustancia, no de forma, variantes significativas, normalmente léxicas, que habría que sopesar a la hora de establecer el texto y, puesto que no podrán determinarse por una selección mecánica, habrá que recurrir entonces a la conjetura y a los dictados del usus scribendi y el propio iudicium del editor. Veamos las principales y más llamativas:

$$
\begin{array}{lll}
\text { v. } 34 & \begin{array}{l}
\text { y llegados } \\
\text { alleguados }
\end{array} & 8 B 386^{*} \text { JM } \\
& \text { allegados } & 83 * I M \text { EM6 PN1 MP2 92VC 95VC 35 CG 40CG }
\end{array}
$$

En la primera lección se trata de la conjunción copulativa $y$ (mejor que pensar en el arcaico adverbio de lugar ý), como revela el signo tironiano de $86 * R L$; de esa lección, que podía estar ya en el subarquetipo $\delta$, salió alleguados, que testifica $82 * J M$, que $83 * I M$ EM6 y demás testimonios dependientes, tal vez por

\footnotetext{
${ }^{3}$ v. 11 qualquier / qualquiera, qualquiere, v. 94 y dellas por calidad / por su calidad, v. 146 trabajosa / trabajada: en el primer caso, el adjetivo indefinido qualquier / qualquiera admite la forma plena o la forma apocopada (aquí la plena regulariza el octosílabo, aunque sería menos correcta, y la apocopada lo hace hipométrico); en el segundo, se incurre en hipermetría; en el tercero, trabajada rima correctamente con celada del v. 149, no así trabajosa; pero los dos sintagmas son característicos de la poesía cancioneril, aquel lo utiliza Mena y este Gómez Manrique, por ejemplo.
} 
contaminación, interpretaron allegados; se produciría, pues, la siguiente secuencia: y llegados / $\tau$ lleguados $>$ alleguados $>$ allegados. En todo caso, hay que reconocer preeminencia, pues, a la lección y llegados.

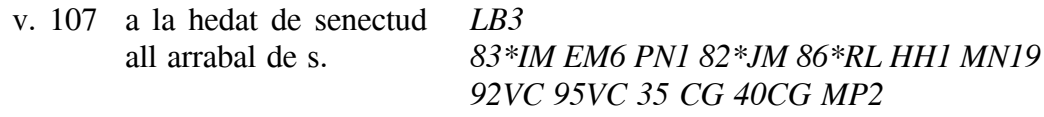

La lección de LB3 es claramente desechable, pues, aparte su singularidad, se trata de un manifiesto error de escritura en el que el copista repite la palabra en rima del v. 91 dellas desfaze la hedat.

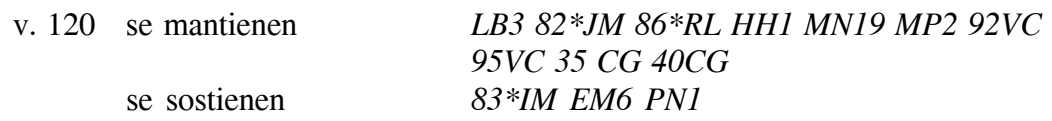

Son lecciones equipolentes, que enfrentan a las dos ramas de la tradición $\alpha$ y $\beta$; más adelante en el v. 354: se sostuvo (Quedando desamparado, / con sus fijos y criados / se sostuvo), común a las dos tradiciones.

$$
\begin{array}{lll}
\text { v. } 139 \text { los plazeres de acá } & \text { LB3 } \\
\text { los deleites } & \text { 83*IM EM6 PN1 82*JM 86*RL HH1 MN19 } \\
& 92 \text { VC 95VC 35CG 40CG MP2 }
\end{array}
$$

La primera lección parecería más correcta para no incurrir en la repetición de palabra (los plazeres de acá / son en que nos deleitamos), aunque va a hablar de plazeres en el v. 145 los plazeres y dulçores; la segunda parecería errónea, porque adelanta repitiendo el verso siguiente, pero también puede entenderse como una licencia poética, una paronomasia: deleites en que nos deleitamos.

$$
\begin{array}{cl}
\text { v. } 173 \text { aunque leemos y oímos } & L B 382 * J M \text { (a. oímos e 1.) } 86 * R L \text { (a. oí- } \\
& \text { mos e 1.) } \\
\text { aunque oímos y leímos } & 83 * I M E M 6 \text { PN1 HH1 MN19 92VC 95VC } \\
& 35 C G 40 C G M P 2
\end{array}
$$

Aparentemente es una variante de lengua consistente en la alternancia de los tiempos verbales, en presente o en pasado; en principio, cualquiera de las dos lecciones hace sentido, pues se refieren al saber de historias romanas leídas y oídas. A las historias troyanas, que acaba de mencionar, se ha referido Manrique en pasado como más alejadas ("Dexemos a los troyanos, / que sus males no los vimos"). Pero las de Roma parecen resultarle más próximas y conocidas, de manera que al emplear ahora el presente está aludiendo a su propio saber y frecuentación de historias romanas, como demostrará unos versos más abajo al sobrepujar a su padre con el canon de emperadores y caudillos romanos. 
v. 174 sus estorias

sus vitorias
LB3 $86 * R L M N 19$ 92VC (historias) 95VC (historias) $35 C G$ 40CG (historias) MP2 (historias)

$83 * I M$ EM6 PN1 82*JM HH1

Es superior la lección primera, pues estorias son el objeto natural de la lectura, que no siempre serían precisamente vitorias.

$\begin{array}{lll}\text { v. } 203 & \text { ropas trenpadas } & \text { LB3 } \\ & \text { ropas chapada } & 83 * I M \text { EM6 PN1 } 86 * R L \text { HHI MN19 92VC } \\ & 95 \mathrm{VC} 35 \mathrm{CG} \text { 40CG MP2 } \\ & \text { ropas japadas } & 82 * J M\end{array}$

En la primera lección, ropas trenpadas está por ropas trepadas, que significa 'guarnecidas con ciertos adornos calados o perforados' $(D C E C H)$. Es término documentado en la época con ese sentido: en las Cuentas de Gonzalo de Baeza, tesorero de Isabel la Católica, se registra varias veces el adjetivo, acompañando a tiras; en el Debate entre Antón de Moros y Gonzalo Dávila, VII, 97 98, aparece el sintagma "sus trepadas / ropas" (edición de Morel-Fatio, 1901); Antonio de Nebrija en su Vocabulario de romance en latín registra: "trepa de vestidura, segmentum [franja, guarnición]". Covarrubias recogerá trepa con el sentido de 'adorno o guarnición de la orilla del vestido'. Por su parte, chapadas alude a los adornos metálicos, y es más usada aplicada a otros objetos, como en el v. 283 "la cava honda, chapada"; en el lenguaje pastoril de Encina, chapada tiene sentido de 'graciosa, alegre, favorecida'. En cuanto a japadas, puede ser interpretación de una grafía*xapadas por chapadas, confundiendo $x$ y $j$.

\section{v. 301 que amigo de amigos $L B 382 * J M$ (de sus) $H H 135 C G 40 C G$ amigo de sus amigos $83 * I M$ EM6 PN1 86*RL MN19 92VC 95VC $M P 2$}

La lección primera, más arraigada en la tradición $\alpha$, parece preferible por el contexto, pues guarda la serie enumerativa que...que (que enemigo de enemigos / que maestre de esforçados), con que se viene ponderando a don Rodrigo, y es paralela y contrapuesta a v. 304 (que enemigo de enemigos); sin el que inicial introductorio se rompe el orden y pierde sentido, aunque se ajustaría más a la métrica, mientras que en el otro caso habría que desechar las sinalefas.

v. 322 en su braço aureliano en su b. un archiano

en la nobleza el troyano $\mathrm{HHI}$

LB3 MN19 $86 * R L$ (abreliano) $82 * J M$ 83*IM EM6 PN1 (un arquiano) 92VC 95VC $35 C G 40 C G$

en sus brazos un troyano $M P 2$ 
Parece evidente que es superior la lección primera aureliano, que algún copista pudo leer también areliano, abreliano; de ese *areliano, por confusión de signos $e>c$ y $l i>h$, pudo salir *archano, archiano, arquiano, que presenta $82 * J M$, así como $83^{*} \mathrm{IM}$ y descendientes. Las lecciones de $H H 1$ y $M P 2$ son innovaciones imaginativas ante la dificultad de entender el nombre. Se refiere, como es sabido, al general y emperador romano Lucio Domicio Aureliano.

\begin{tabular}{|c|c|c|}
\hline v. 323 & marco tulio en la verdat & $\begin{array}{l}\text { LB3 } 82 * J M \quad 83 * I M \text { EM6 PN1 } 92 V C^{4} \\
95 V C \text { 35CG 40CG }\end{array}$ \\
\hline & atilit & $86 * R L H H 1$ (autilio) MN19 \\
\hline
\end{tabular}

En este caso, la lección correcta es la segunda, pues el mencionado es Marco Atilio, que conocería bien Manrique a través de la Carta sobre el menosprecio del mundo de San Euquerio, que gozó de gran difusión en la Edad Media y que imita en la c. VII (Lida de Malkiel, 1977: 145 178). También pudieron ser fuente los Proverbios del marqués de Santillana, cuyas coplas 69 y 70 ensalzan a Marco Atilio como modelo de verdad (Marco Atilio non dubdando / que muriesse / si a Cartagena bolviesse, / observando / la verdad, non dilatando / se bolvió, / donde luego padesçió / retornando). La otra es una clara lectio facilior: Marco Tulio por Marco Atilio. Es un error común en los planos altos de $\alpha$ y $\beta$, que podría pronosticar un arquetipo $x$. Lo repararía tardíamente por conjetura $86^{*} \mathrm{RL}$ y lo transmitiría seguramente a $\varepsilon$.

\section{v. 385 después de puesta la vida LB3 $82 * J M 86 * R L$ 92VC 95VC $35 C G$ $40 C G$ después que puso la vida $83 * I M$ EM6 HH1 MN19}

Es un cambio de forma y tiempo verbal para evitar repetición de sonidos despues depuesta; quizá suponga una corrección embellecida de un texto sobre otro.

$$
\begin{array}{r}
\text { v. } 406 \text { fazedla de la virtud } \\
\text { esfuércese la virtud }
\end{array}
$$

$L B 3$

$83 * I M$ EM6 PN1 $82 * J M \quad 86 * R L H H 1$ MN19 92VC 95VC 35CG 40CG MP2 (e. vuestra virtud)

El sentido de los versos ("y pues de vida y salud / fezistes tan poca cuenta, / por la fama, / fazedla de la virtud / para sofrir esta afrenta / que vos llama") viene a ser: 'pues no hicisteis cuenta (no os importó) de la vida ni la salud por

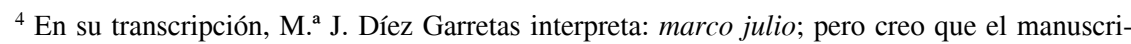
to lo que presenta es marco tulio, pues es el mismo signo para la $t$ - que el que emplea en el $\mathrm{v}$. 320 para tito, distinto del empleados en v. 314 para julio.
} 
conseguir la fama, hacedla ahora de la virtud para afrontar la muerte'. En realidad, la lección de LB3 hace más sentido y no fuerza el orden gramatical.

$$
\begin{array}{lll}
\text { v. } 417 \text { no es eternal / ni duradera } & L B 3 \\
\text { no es eternal / ni verdadera } & 83 * I M E M 682 * J M 86 * R L M N 19 P N 1 \text { (om. } \\
& \text { ni) MP2(om. ni) 92VC 95VC 35CG 40CG }
\end{array}
$$

Se refieren ambas lecciones a la vida de la fama (v. 415 vida de honor); en principio, son lecciones equipolentes, pero duradera se corresponde mejor con eternal y con los adjetivos de temporalidad que emplea en toda la estrofa: v. 419 temporal, v. 420 perescedera.

$$
\begin{array}{lll}
\text { v. } 443 \text { que la vida verdadera } & \text { LB3 } \\
\text { que esta otra vida tercera } & 83 * I M \text { EM6 PN1 82*JM 86*RL MN19 } \\
& 92 \text { VC 95VC 35CG 40CG } \\
& \text { que la otra vida eterna } & \text { MP2 }
\end{array}
$$

LB3 no ha hablado hasta aquí de la vida verdadera, mientras que los demás sí lo han hecho en el v. 417, es por lo que no tiene necesidad de introducir un nuevo adjetivo que califique la vida perdurable que ha ganado el maestre; nótese que $M P 2$ tampoco menciona ninguna vida tercera.

$$
\begin{array}{lll}
\text { v. } 444 \text { cobraréis } & \text { LB3 } \\
\text { ganaréis } & 83 * I M \text { EM6 PN1 } 82 * J M \quad 86 * R L \text { MN19 } \\
& 92 \text { VC 95VC 35CG 40CG MP2 }
\end{array}
$$

El verbo preferido en todo el texto de las Coplas es ganar; sólo en v. 378 aparece cobrar: "las cobró [sus villas y sus tierras]"; LB3 lo vuelve a utilizar aquí para evitar la repetición del v. 437: "que en este mundo ganastes".

$$
\begin{array}{lll}
\text { v. } 471 \text { conservados } & L B 382 * J M \quad 86 * R L \text { MN19 } 95 V C M P 2 \\
& 35 C G 40 C G \\
& \\
\text { olvidados } & 83 * I M \text { EM6 PN1 92VC }
\end{array}
$$

Es una variante que enfrenta claramente a las dos ramas de la tradición $\alpha$ y $\beta$ y refleja la fuerte contaminación del texto sucesivo (nótese la diferencia entre 92VC y 95VC, casi siempre coincidentes en sus lecciones, como ya dijimos). Más conforme a la documentación de época, a las prescripciones de las artes moriendi, que en estos versos tiene presentes Manrique, y a las actitudes de muertes memorables, en las que la conservación del juicio es elemento fundamental de la buena muerte, resulta la lección conservados de $\alpha$ (Conde, 2009: 59-84).

A la vista de lo expuesto, considero que habría que dar prevalencia a las siguientes variantes, más propias de la rama $\alpha$, que en su mayoría presenta de 
manera singular $L B 3$ y, en consecuencia, corregir en el texto más habitualmente aceptado de las Coplas (en su vulgata, diríamos): v. 34 y llegados y no allegados; v. 121 se mantienen y no se sostienen; v. 140 los plazeres y no los deleites; v. 173 aunque leemos y oímos y no aunque oímos y leímos; v. 175 sus estorias y no sus vitorias; v. 203 ropas trenpadas y no ropas chapadas; v. 204 que traían y no que vestían; v. 301 que amigo de (sus) amigos y no amigo de sus amigos; v. 406 fazedla de la virtud y no esfuércese la virtud; v. 417 ni duradera y no ni verdadera; v. 443 que la vida verdadera y no que esta otra vida tercera; v. 444 cobraréis y no ganaréis; v. 471 conservados y no olvidados.

Habría que desechar, en cambio, errores manifiestos de esa misma procedencia, como: v. 107 a la hedat por al arrabal; v. 323 marco tulio por Marco Atilio; v. 353 con sus fijos y criados por con hermanos y criados; v. 385 después de puesta la vida por después que puso la vida; v. 419 que la otra infernal por que la otra temporal.

Como decimos, son variantes en su mayoría procedentes del cancionero Egerton, testimonio bien situado en los niveles altos del stemma, relativamente próximo al original. Se trata de un cancionero, según los estudios de Dorothy Severin y Vicente Beltrán citados, copiado en los últimos años de la década de 1470 y los primeros de la siguiente en la corte de Navarra desde un modelo castellano. Un cancionero de poesía consolatoria y didáctico moral, que, como vemos, independientemente de sus numerosos errores, ofrece en sus variantes un texto sobrio y preciso, menos ornamentado que el que presenta la copia inserta en los cancioneros de fray Íñigo de Mendoza (sobre todo $83 * I M$ ), con lecciones quizá más sugestivas (allegados, deleites, oímos y leímos, sus vitorias, chapadas, vestían, vida tercera), pero tal vez menos auténticas.

\section{BIBLIOGRAFÍA}

Beltrán, Vicenç (2011): "Notas al Cancionero Ms. Egerton 939 (LB3)”, en Andrea Baldissera, Giuseppe Mazzocchi y Paolo Pintacuda (eds.), Ogni onda si rinnova. Studi di ispanistica offerti a Giovanni Caravaggi, Pavía, Ibis, I, pp. 159-175.

Beltrán, Vicente (1987): "La transmisión textual de las Coplas manriqueñas (1480- 1540)", Incipit, 7, pp. 95-117.

Beltrán, Vicente (ed.) (1988): Jorge Manrique, Poesía completa, 2. ed., Barcelona, Planeta.

Beltrán, Vicente (ed.) (1993): Jorge Manrique, Poesía, Barcelona, Crítica.

Beltrán, Vicente (ed., estudio y notas) (2013): Jorge Manrique, Poesía, Madrid/Barcelona, Real Academia Española/Galaxia Gutenberg-Círculo de Lectores.

Caravaggi, Giovanni (ed.) (1984): Jorge Manrique, Poesía, Madrid, Taurus.

Cerrón Puga, M. ${ }^{a}$ Luisa (2012): "Los libros del Duque: textos españoles en el Fondo Urbinate de la Alessandrina", en Patrizia Botta (coord.), Rumbos del hispanismo en el umbral del Cincuentenario de la Asociación Internacional de Hispanistas. Medieval, Aviva Garribba (ed.), Roma, Bagatto Libri, 2012, vol. II, pp. 290-300.

Conde, J. Carlos (2009): "Conservados / olvidados: una copla de Manrique y los límites del método neolachmanniano", Incipit, 29, pp. 59-84. 
DCECH = Corominas Joan y José A. Pascual (1980-1991): Diccionario crítico etimológico castellano e hispánico, 6 vols., Madrid, Gredos.

Díez Garretas, M. ${ }^{a}$ Jesús (2010): "El cancionero MN46 (BNE, Ms, 18183): del impreso al manuscrito", en Actas del XIII Congreso Internacional de la Asociación Hispánica de Literatura Medieval, Valladolid, Universidad de Valladolid, pp. 697-717.

Dutton, Brian (1990-1991): El cancionero del siglo XV, c. 1360-1520, 7 vols., Biblioteca Española del s. XV, Salamanca, Universidad de Salamanca.

Fernández Álvarez, Benigno (1904): "Incunables españoles en la Biblioteca escurialense”, $L a$ Ciudad de Dios, 63, pp. 586-593.

Fernández Álvarez, Benigno (1911): "Incunables españoles de la Biblioteca del Escorial”, La Ciudad de Dios, 86, pp. 55-70.

Garcia, Michael (1978-1980): "Le Chansonnier d'Oñate y Castañeda", Mélanges de la Casa de Velázquez, 14, pp. 107-142; 15, pp. 207-239; y 16, pp. 141-149.

Gómez Moreno, Ángel (ed.) (2000): Jorge Manrique, Poesía completa, Madrid, Alianza Editorial.

Hock, David (1987): “An Idiosyncratic Manuscript Copy of Jorge Manrique's Coplas por la muerte de su Padre (Lisbon, Bibl. Nac., cod. 11353)", Scriptorium, 41, pp. 237-254.

Labrador, José J., C. Ángel Zorita y Ralph A. Difranco (1985): "Cuarenta y dos, no cuarenta coplas en la famosa elegía manriqueña”, Boletín de la Biblioteca Menéndez Pelayo, 61, pp. 37-95.

Labrador, José J., C. Ángel Zorita y Ralph A. Difranco (eds.) (1986): Cancionero de poesías varias. Manuscrito No. 617 de la Biblioteca Real de Madrid, Madrid, El Crotalón.

Lida de Malkiel, M. ${ }^{a}$ Rosa (1977): "Una copla de Jorge Manrique y la tradición de Filón en la literatura española”, en Estudios sobre la literatura española del siglo XV, Madrid, José Porrúa Turanzas, pp. 145178.

Morel-Fatio, Alfred (1901): "Le Débat entre Antón de Moros et Gonzalo Dávila", Romania, 30, pp. 49-64.

Morrás, María (ed.) (2003): Jorge Manrique, Poesía, Madrid, Castalia.

Palumbo, Pietro (1983): "L'ordine delle strofe nelle Coplas por la muerte de su padre di Jorge Manrique", Medioevo Romanzo, 8, pp. 193215.

Pérez Gómez, Antonio (1958): Tercera Floresta de Incunables, Incunables Poéticos Castellanos, VIII, Cieza.

Pérez Gómez, Antonio (1975): Fray Íñigo de Mendoza, Vita Christi fecho por coplas. Incunables Poéticos Castellanos, XIV, Cieza.

Pérez Priego, Miguel Ángel (ed.) (1990): Jorge Manrique, Poesías completas, Colección Austral, A 152, Madrid, Espasa-Calpe.

Senabre, Ricardo (1983): "La primera edición de las Coplas de Jorge Manrique", en Serta Philologica F. Lázaro Carreter, Madrid, II, pp. 509517.

Serrano de Haro, Antonio (ed.) (1986): Jorge Manrique, Obras, Madrid, Alhambra.

Severin, Dorothy S. (2000): Two Spanish Songbooks. The 'Cancionero Capitular de la Colombi$n a$ ' (SV2) and the 'Cancionero de Egerton' (LB3), Liverpool, Liverpool University Press/Institución Colombina.

Severin, Dorothy S. y Michel Garcia (eds.) (1990): El Cancionero de Oñate-Castañeda, Madison, Hispanic Seminary of Medieval Studies.

Uhagón, Francisco R. de (1900): "Un cancionero del siglo XV, con varias poesías inéditas", Revista de Archivos Bibliotecas y Museos, 4, pp. 321-338, 390-403 y 516-535.

Fecha de recepción: 4 de octubre de 2016

Fecha de aceptación: 14 de diciembre de 2016 